\title{
Controlled Noise Seismology
}

Sherif M. Hanafy*, Abdullah AlTheyab, and Gerard T. Schuster

King Abdullah University of Science and Technology (KAUST), Thuwal, Saudi Arabia

\section{Summary}

We use controlled noise seismology (CNS) to generate surface waves, where we continuously record seismic data while generating artificial noise along the profile line. To generate the CNS data we drove a vehicle around the geophone line and continuously recorded the generated noise. The recorded data set is then correlated over different time windows and the correlograms are stacked together to generate the surface waves. The virtual shot gathers reveal surface waves with moveout velocities that closely approximate those from active source shot gathers.

\section{Introduction}

Ambient noise seismology was developed by seismologists (Shapiro et al., 2005; Lin et al., 2008; Lin et al., 2009; Lin and Ritzwoller, 2011; Lin et al., 2013) to create virtual shot gathers from ambient noise recordings. The location of each virtual shot is at the recording geophone. For earthquakes and microseisms, the typical recording time to transform ambient noise into useful shot gathers ranges from several months to several years. The surface waves recorded in the virtual shot gathers are often dominated by the fundamental mode of the Rayleigh wave and can be inverted for the S-velocity distribution (Aki and Richards, 1980; Lin et al., 2013).

It was recently shown that the $\mathrm{S}$-velocity tomogram inverted from passive data can be used to assess the near surface for drilling hazards or statics (de Ridder et al., 2014). However, the virtual shot gathers require a long period of recording time (more than several weeks) in order to create usable shot gathers. To overcome this long recording time we test the possibility of using a controlled noise source (CNS) to create the virtual shot gathers. In our test example we employ a wooden beam dragged behind a truck that circles the array for 150 minutes.

In the recording seismograph, we activate the continuous recording options so that that total recorded time is 150 minutes without any time gaps. The data are recorded in small files, each one is $32 \mathrm{~s}$ long with $2 \mathrm{~s}$ overlaps between files, in processing time we remove the $2 \mathrm{~s}$. The time interval in the recorded data is $1 \mathrm{~ms}$.

\section{Theory}

The controlled noise is created by a controlled noise source. If the noise source wavelet is a random time series and surrounds the array then Wapenaar (2004) shows that

$$
\operatorname{Im}[G(\boldsymbol{A} \mid \boldsymbol{B})]=k<P(\boldsymbol{A})^{*} P(\boldsymbol{B})>
$$

where $P(\boldsymbol{A})$ is the passive data recorded at receiver $\boldsymbol{A}, P(\boldsymbol{B})$ is the passive data recorded at receiver $\mathrm{B}, k$ is the wavenumber, <> denotes averaging, and $G(\boldsymbol{A} \mid \boldsymbol{B})$ is the virtual shot gather with a virtual shot at $\boldsymbol{B}$ and a receiver at A (Schuster, 2009, chapter 10).

In practice, the virtual shot gather is calculated by correlating time-windowed traces recorded at $\boldsymbol{A}$ and $\boldsymbol{B}$, and averaging the correlograms over many time windows. The energy in each time window should be normalized, and the window length should be at least ten times the time of the latest arriving event expected from the data.

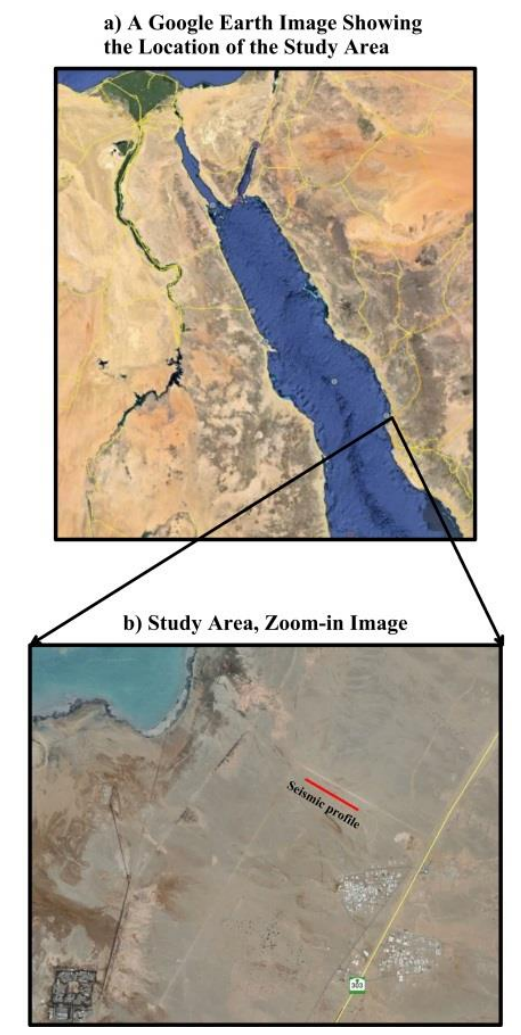

Figure 1: (a) A google earth image shows the location of the study area located at the western side of Saudi Arabia along the Red Sea coastline. (b) A zoom view shows the location of the $600 \mathrm{~m}$ seismic profile. 


\section{Controlled Noise Seismology}

\section{Numerical Tests}

The CNS technique is tested on 2D field data recorded over a normal fault known as Qademah fault. The study area is located along the western side of Saudi Arabia near the Red Sea Coast (Figure 1).

\section{CNS Data Recording}

We used 2 types of receivers to record this data set; the first type has a natural peak-frequency of $40 \mathrm{~Hz}$ while the second one is $4.5 \mathrm{~Hz}$ (Figure 2) with $5 \mathrm{~m}$ geophone intervals. The receivers are arranged in alternating order as shown in Figure 2. Three wooden bars and two tires were tied behind the truck to create additional noise. This controlled noise source is a practical alternative to Snieder's noise truck with Powerpoint wheels (see inset in Figure 3).

To generate the CNS signals we drove a pickup vehicle for 150 minutes around the profile (Figure 3). The driving speed ranged between 20 and $25 \mathrm{~km} / \mathrm{h}$. To record the noise signals we used a geometrics seismograph system with a sample interval of $1 \mathrm{~ms}$ and the "continuous recording" option in the acquisition software is activated. Figure 4 shows a sample of the recorded data.

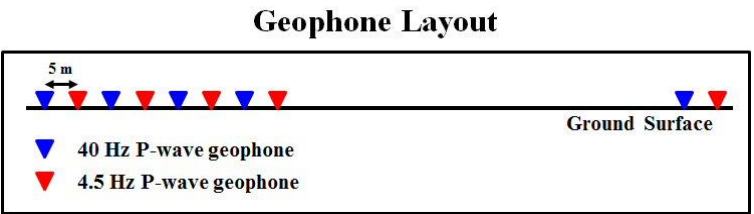

Figure 2: The receiver layout. We used two types of receivers, the first has a peak frequency of $4.5 \mathrm{~Hz}$ and the second is $40 \mathrm{~Hz}$.

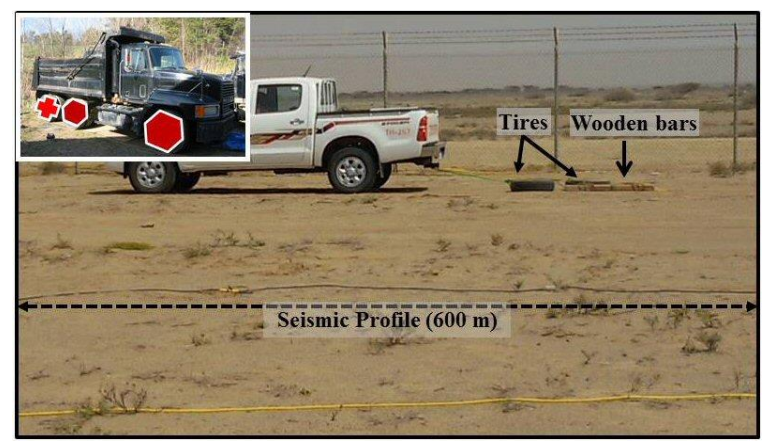

Figure 3: A photo taken during data survey. We used 2 old tires and 3 wooden bars as the noise makers, the receivers were continuously recording for 150 minutes. Snieder's Powerpoint wheel truck as an alternative noise maker is shown in the small frame.
The recorded CNS data is correlated and stacked as described in equation (2). Here, we divided each 150minute long trace into 9 time windows, we correlated each windowed trace with a master trace and then stacked the results together. We did not use any kind of normalization, gain, or filter before the correlation + stacking process.

a) Controlled Noise Seismic data, $4.5 \mathrm{~Hz}$ Geophones

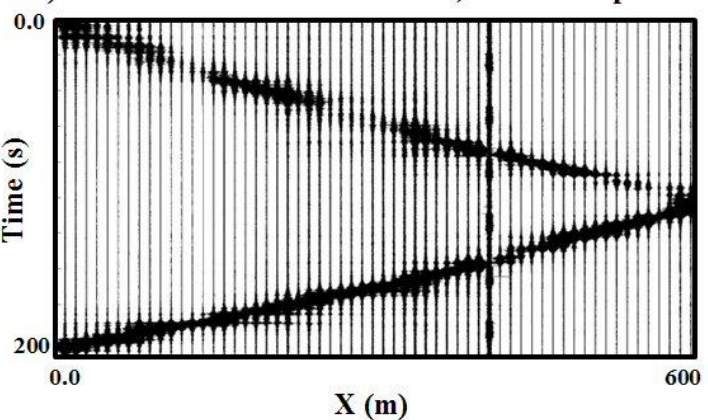

b) Controlled Noise Seismic data, $40 \mathrm{~Hz}$ Geophones

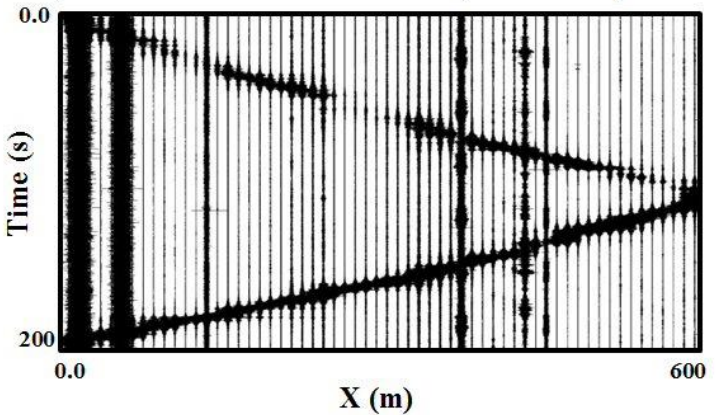

Figure 4: Two samples of the recorded data for receivers with peak frequencies of a) $4.5 \mathrm{~Hz}$ and b) $40 \mathrm{~Hz}$. No normalization, gain, or filter are applied to this data.

The virtual shot gathers are band-pass filtered using a frequency band of 5, 10, 30, $50 \mathrm{~Hz}$. Figure 5a shows the virtual shot gather after band-pass filtering for a master trace located at the center of the receiver line; here we only used the $4.5-\mathrm{Hz}$ receivers. Figure $5 \mathrm{c}$ is similar to Figure $5 \mathrm{a}$, except only the $40-\mathrm{Hz}$ receivers are used. Coherent signals are shown in the causal and acausal parts of the final virtual shot gathers (Figures 5a and 5c). These coherent signals are corresponding to the surface waves.

To compare the virtual shot gathers with ground truth, we recorded active source data set using a $40 \mathrm{~kg}$ mechanical weight drop. Figures $5 \mathrm{~b}$ and $5 \mathrm{~d}$ show two examples of the active-source shot gathers where the source is located at the center of the profile $(X=300 \mathrm{~m})$ and receivers are at both sides, where Figure $5 \mathrm{~b}$ and $5 \mathrm{~d}$ the virtual shot gathers for the $4.5 \mathrm{~Hz}$ and $40 \mathrm{~Hz}$ receivers, respectively. 


\section{Controlled Noise Seismology}

Comparing Figure 5a with Figure $5 \mathrm{~b}$ and Figure $5 \mathrm{c}$ with Figure 5d show an excellent resemblance between the actual and virtual surface waves. We also note that the 4.5 $\mathrm{Hz}$ receivers produce a virtual shot gather with higher signal to noise ratio relative to the virtual shot gather generated from the $40 \mathrm{~Hz}$ receivers. This is because the CNS technique generates low-frequency signals, and the $4.5-\mathrm{Hz}$ receivers are able to more faithfully record low frequency data than the $40-\mathrm{Hz}$ receivers.

A final test is assess the amount of time needed to generate virtual shot gathers with good signal-to-noise ratio. Figure 6 shows the same as Figure 5, except only 10 minutes of CNS (Figures 6a and 6b) and 30 minutes of CNS (Figures $6 \mathrm{c}$ and $6 \mathrm{~d}$ ) were used to generate the virtual shot gather. It is obvious that in case of 4.5 receivers, only 10 minutes of CNS data were sufficient to get a shot gather that can be inverted for the S-velocity.

\section{Conclusions}

Virtual shot gathers are created from controlled noise sources for our test site. Only 150 minutes of recording time are needed to generate virtual shot gathers with surface waves that resemble those from an active source. Body-wave reflections are not visible in the virtual shot gathers, but the surface waves can be inverted to give the 2D S-velocity tomogram. The tomogram can be used to assess drilling hazards and solutions to statics.

For an exploration survey, it might be possible to drive the vibroseis units around the recording array so that virtual shot gathers can be created for shots at each of the geophones. With dense recording arrays, this means that an order-of-magnitude more shot gathers could be generated and used for S-velocity inversion compared to a conventional survey. The main benefit of controlled noise seismology is that it requires several orders-of-magnitude less recording time than ambient noise seismology. One of the earlier proponents of controlled noise source is Roel Snieder.

Future research will aim at determining the optimal type and duration of controlled noise sources that are needed to produce usable virtual CSGs for a recording array with a given diameter.

\section{Acknowledgements}

This publication is based upon work supported by the KAUST Office of Competitive Research Funds (OCRF) under Award No.OCRF-2014-CRG3-62140387/ORS\#2300

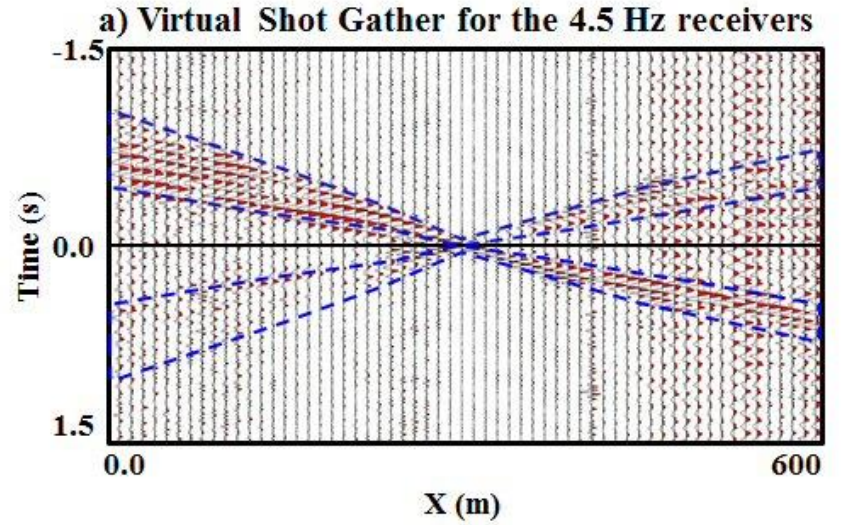

b) Active Shot Gather for the $4.5 \mathrm{~Hz}$ receivers
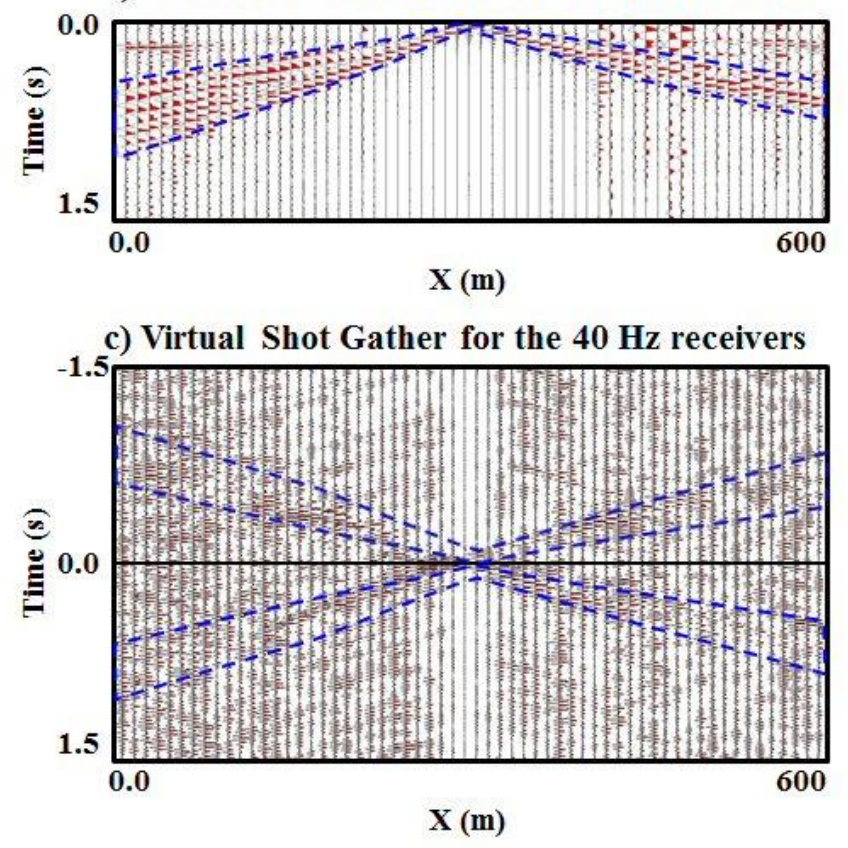

d) Active Shot Gather for the $40 \mathrm{~Hz}$ receivers

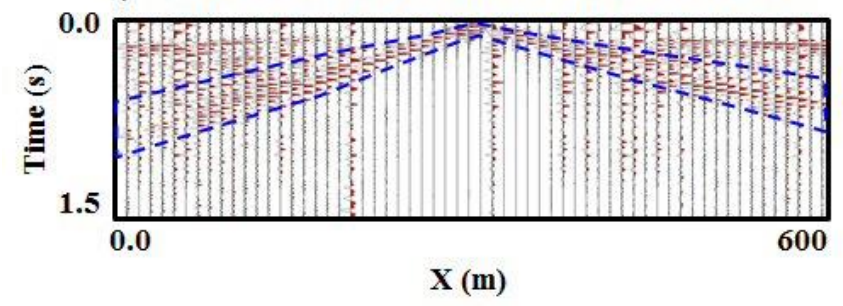

Figure 5: a) Controlled noise shot gather for the $4.5 \mathrm{~Hz}$ receivers and b) the active source shot gather for the same receivers. c) Controlled noise shot gather for the $40 \mathrm{~Hz}$ receivers and d) the active shot gather for the same receivers. The CNS shot gather was computed from 150 minutes of noise. 


\section{Controlled Noise Seismology}

a) Virtual Shot Gather for the $4.5 \mathrm{~Hz}$ receivers from 10 minutes of CNS data

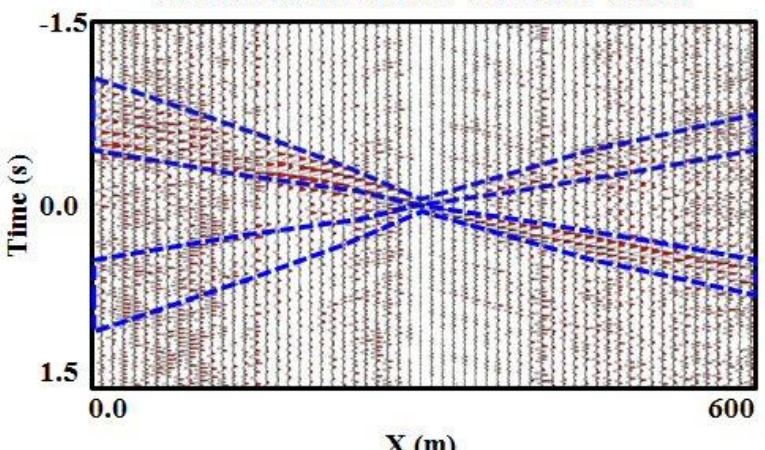

c) Virtual Shot Gather for the $4.5 \mathrm{~Hz}$ receivers from 30 minutes of CNS data

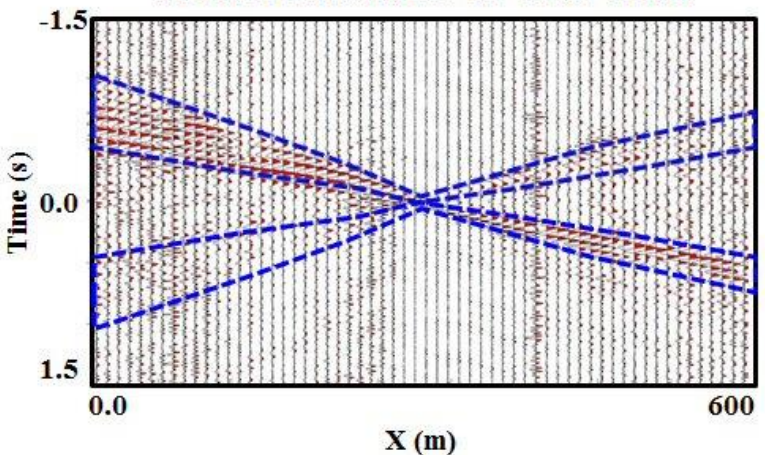

b) Virtual Shot Gather for the $40 \mathrm{~Hz}$ receivers from 10 minutes of CNS data

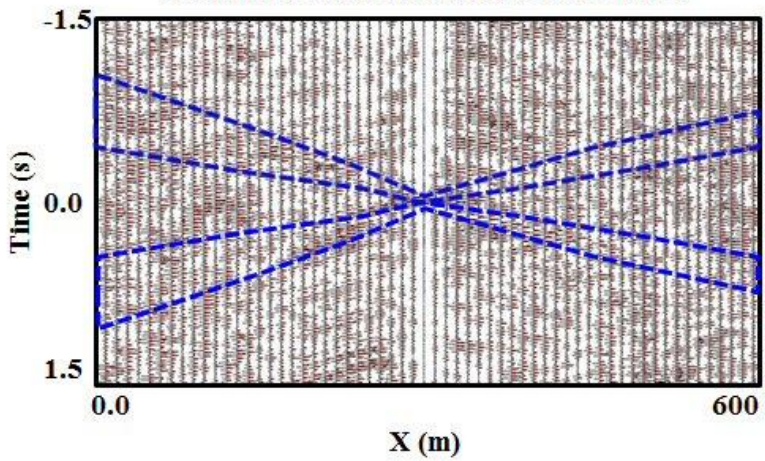

d) Virtual Shot Gather for the $40 \mathrm{~Hz}$ receivers from 30 minutes of CNS data

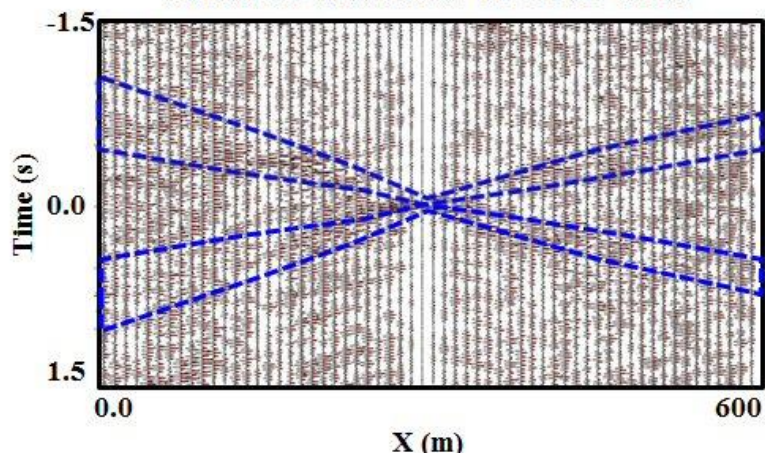

Figure 6: Same as Figure 5, except only 10 minutes of CNS data were used to compute the virtual shot gather for the $4.5-\mathrm{Hz}$ and $40-\mathrm{Hz}$ receivers shown on a) and b), respectively. c) and d) show the virtual shot gather for the $4.5-\mathrm{Hz}$ and the $40-\mathrm{Hz}$ receivers, respectively, using 30 minutes of CNS data. 


\section{EDITED REFERENCES}

Note: This reference list is a copyedited version of the reference list submitted by the author. Reference lists for the 2015 SEG Technical Program Expanded Abstracts have been copyedited so that references provided with the online metadata for each paper will achieve a high degree of linking to cited sources that appear on the Web.

\section{REFERENCES}

Aki, K., and P. Richards, 1980, Quantitative Seismology: W. H. Freeman and Company.

de Ridder, S. A. L., B. L. Biondi, and R. G. Clapp, 2014, Time-lapse seismic noise correlation tomography at Valhall: Geophysical Research Letters, 41, no. 17, 6116. http://dx.doi.org/10.1002/2014GL061156.

Lin, F., M. P. Moschetti, and M. H. Ritzwoller, 2008, Surface wave tomography of the western United States from ambient seismic noise: Rayleigh and Love wave phase velocity maps: Geophysical Journal International, 173, no. 1, 281-298. http://dx.doi.org/10.1111/j.1365-246X.2008.03720.x.

Lin, F., and M. H. Ritzwoller, 2011, Helmholtz surface wave tomography for isotropic and azimuthally anisotropic structure: Geophysical Journal International, 186, no. 3, 11041120. http://dx.doi.org/10.1111/j.1365-246X.2011.05070.x.

Lin, F., M. H. Ritzwoller, and R. Snieder, 2009, Eikonal Tomography: Surface wave tomography by phase-front tracking across a regional broad-band seismic array: Geophysical Journal International, 177, no. 3, 1091-1110. http://dx.doi.org/10.1111/j.1365-246X.2009.04105.x.

Lin, F. C., D. Li, R. W. Clayton, and D. Hollis, 2013, High-resolution 3D shallow crustal structure in Long Beach, California: Application of ambient noise tomography on a dense seismic array: Geophysics, 78, no. 4, Q45-Q56. http://dx.doi.org/10.1190/geo2012-0453.1.

Schuster, G. T., 2009, Seismic Interferometry: Cambridge Press. http://dx.doi.org/10.1017/CBO9780511581557.

Shapiro, N., and M. Campillo, L. Stehly, and M. Ritzwoller, 2005, High-resolution surface wave tomography from ambient noise: Nature, 1615-1618.

Wapenaar, K., 2004, Retrieving the elastodynamic Green's function of an arbitrary inhomogeneous medium by crosscorrelations: Physical Review Letters, 93, no. 25, 254301. http://dx.doi.org/10.1103/PhysRevLett.93.254301. 